\title{
The new era of RNA modification
}

\author{
JOHN KARIJOLICH ${ }^{1}$ and YI-TAO YU ${ }^{2}$ \\ ${ }^{1}$ Department of Plant and Microbial Biology, University of California, Berkeley, California 94720-3370, USA \\ ${ }^{2}$ Department of Biochemistry and Biophysics, Center for RNA Biology, University of Rochester School of Medicine and Dentistry, \\ Rochester, New York 14642, USA
}

Since the inception of the journal RNA in 1995, we have witnessed significant advances in a number of areas of RNA research. Among them is the area of RNA modification. In the mid-1990's, a "sno" storm hit the RNA field, leading to the identification of a large number of small nucleolar RNAs, known as box H/ACA and box C/D snoRNAs. The discovery of these RNAs generated a great deal of new interest in snoRNA research, which, in turn, led to a breakthrough in the area of RNA modification: It turned out that box C/D and box H/ACA RNAs exist in the cell as RNA-protein complexes, or snoRNPs (each snoRNA is complexed with four common proteins; Nop1/fibrillarin, Nop56, Nop58, and Snu 13 for box C/D RNPs, and Cbf5/NAP57/Dyskerin, Nhp2, Gar1, and Nop10 for box H/ACA RNPs), and that the RNA component (box C/D RNA or box H/ACA RNA) of each RNP complex functions as a guide that directs, in a site-specific manner, rRNA 2'-O-methylation or pseudouridylation, respectively.

The discovery of RNA-guided RNA modification mechanisms sparked a new wave of research interest in RNA modification, yielding fruitful results. For instance, it has been shown that the RNA-guided (or snoRNP-catalyzed) mechanisms, which modify rRNA in the nucleolus, can be applied to spliceosomal snRNA modification as well. Soon after, it was found that the guide RNAs that direct snRNA modifications were co-localized with their substrate snRNAs in the subnuclear domains known as Cajal bodies. In addition, a number of new stand-alone protein enzymes, responsible for pseudouridylation and 2'-O-methylation (and other types of modifications) of stable RNAs (tRNA in particular), have also been identified. Furthermore, crystal structures of the stand-alone protein enzymes as well as substrate-bound box $\mathrm{C} / \mathrm{D}$ and box H/ACA RNPs are now available, providing a detailed molecular understanding of site-specific $2^{\prime}-\mathrm{O}-$ methylation and pseudouridylation.

Significant progress has also been made towards understanding the function of modified nucleotides. Because

\footnotetext{
Corresponding author: yitao_yu@urmc.rochester.edu

Article and publication date are at http://www.rnajournal.org/cgi/doi/ 10.1261/rna.049650.115. Freely available online through the RNA Open Access option.
}

rRNAs and spliceosomal snRNAs are enriched with both 2'-O-methylated residues and pseudouridines, these two modifications have been extensively studied. Experimental data accumulated over the past two decades have indicated that modified nucleotides are concentrated in strategically important regions of rRNAs and spliceosomeal snRNAs (at the primary, secondary, and tertiary structural levels), and that they are indeed functionally important for rRNA biogenesis, protein translation, and pre-mRNA splicing. And even though in eukaryotes, tRNA $2^{\prime}$-O-methylation and pseudouridylation (and tRNA modification in general) are not catalyzed by RNA-guided mechanisms (instead, these modifications are catalyzed by stand-alone protein enzymes), the mechanisms and functions of tRNA modification have attracted enormous attention as well, generating a number of interesting findings. For instance, it has been reported that modified nucleotides in the anticodon loop of tRNA contribute significantly to translation fidelity. Additionally, it has been shown that newly synthesized tRNAi ${ }^{\text {Met }}$ lacking methylation at one position (position 58) is subject to degradation in the nucleus. Many other modified nucleotides also play an important role in maintaining tRNA structure and stability. Indeed, removal of some specific modifications results in rapid tRNA decay.

Another significant advance in the study of RNA modification was the realization that modified nucleotides can be inducibly introduced into an RNA. Prior to this, it was widely assumed that RNA modifications (pseudouridylation and 2'$\mathrm{O}$-methylation in particular) occur constitutively. This assumption was recently challenged by the observation that under certain stress conditions, pseudouridylation can be induced in spliceosomal snRNAs at novel positions. The sequences of inducible sites are imperfect and more flexible than those of constitutively pseudouridylated sites, suggesting that there may be a large number of such loosely defined sites and that induced pseudouridylation is a widespread phenomenon.

Recently, research into RNA modification has extended its scope to include mRNA modification as well. Remarkably,

(C) 2015 Karijolich and $\mathrm{Yu}$ This article, published in $R N A$, is available under a Creative Commons License (Attribution-NonCommercial 4.0 International), as described at http://creativecommons.org/licenses/by-nc/4.0/. 
targeted pseudouridylation at the first position of stop codons results in nonsense suppression both in vitro and in vivo. It appears that pseudouridylated stop codons reject the release factor and promote unusual codon-anti-codon interactions in the decoding center. This finding suggests that mRNA modification (pseudouridylation in particular) possibly represents yet another mechanism of generating protein diversity. It has also been reported that incorporation of pseudouridine into mRNA results in increased translation efficiency and reduced RNA-elicited innate immune responses. Most recently, a transcriptome-wide pseudouridine-seq approach has been developed, allowing the identification of a large number of naturally occurring mRNA pseudouridines in both yeast and mammalian cells. These pseudouridines appear to be scattered throughout the mRNA, including the coding region and the $5^{\prime}$ and $3^{\prime}$ UTRs, and many of them are stress-inducible.

RNA modifications are not limited to pseudouridylation and 2'-O-methylation; both tRNA and mRNA contain other types of modifications, which have also been extensively studied over the past 20 years. In particular, $\mathrm{N}^{6}$-methyladenosine $\left(\mathrm{m}^{6} \mathrm{~A}\right)$ in mRNA has attracted an enormous amount of attention. Recent work has demonstrated that the $\mathrm{m}^{6} \mathrm{~A}$ modification is reversible (a set of de-methylation enzymes have been identified), suggesting a regulatory role for this modified nucleotide. Indeed, it has recently been reported that $\mathrm{m}^{6} \mathrm{~A}$ plays a role in mRNA stability and the maintenance of embryonic stem cell identity.

While there have been significant advances in the area of RNA modification research in the past two decades, many questions remain. Among the most interesting are those regarding mRNA modification. For instance, what regulatory roles do mRNA modifications play? Given that pseudouridines are present in both coding and untranslated regions, it is possible that they affect coding specificity, translation efficiency, mRNA stability, or even all three. Experimental verification of any of these potential functions would constitute a great leap forward in the study of RNA modification. Another important question is: how are mRNA modifications induced by stress and what functional roles do these induced modifications play? It appears that the modifying enzymes are available to the inducible substrates (imperfect sequences) under both normal and stress conditions, but only under stress conditions are the inducible substrates modified. Detailed analyses, directed towards understanding the mechanisms and functions of induced modifications, are highly important.

As seen in the past 20 years, the pace of RNA modification research has quickened, and momentum is clearly building in the field. With the help of modern technologies, a new, more thorough picture of RNA modification (especially mRNA modification) is expected to emerge. The era of RNA modification research has arrived. 

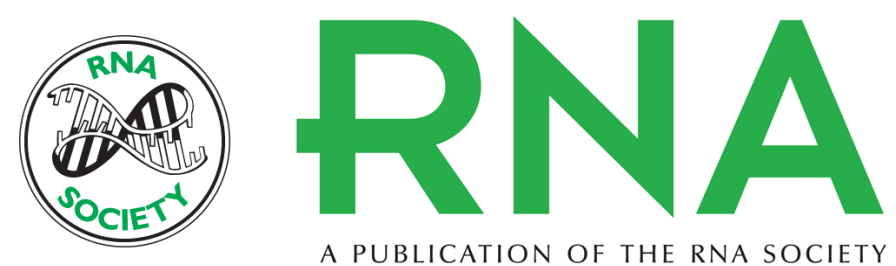

A PUBLICATION OF THE RNA SOCIETY

\section{The new era of RNA modification}

John Karijolich and Yi-Tao Yu

RNA 2015 21: 659-660

Open Access Freely available online through the RNA Open Access option.

Creative This article, published in $R N A$, is available under a Creative Commons License

Commons (Attribution-NonCommercial 4.0 International), as described at

License http://creativecommons.org/licenses/by-nc/4.0/.

Email Alerting Receive free email alerts when new articles cite this article - sign up in the box at the Service top right corner of the article or click here.

To subscribe to $R N A$ go to:

http://rnajournal.cshlp.org/subscriptions

(C) 2015 Karijolich and Yu; Published by Cold Spring Harbor Laboratory Press for the RNA Society 